\title{
Perspectives of Clinicians at Skilled Nursing Facilities on 30-Day Hospital Readmissions: A Qualitative Study
}

\author{
Bennett W. Clark, MD ${ }^{1 *}$, Katelyn Baron, MSIOP², Kathleen Tynan-McKiernan, RN, MSN, ${ }^{2}$ \\ Meredith Campbell Britton, $\mathrm{LMSW}^{3}$, Karl E. Minges, $\mathrm{PhD}^{3}$, Sarwat I. Chaudhry, MD
}

\begin{abstract}
${ }^{1}$ Department of Internal Medicine, Johns Hopkins University School of Medicine, Baltimore, Maryland; ${ }^{2}$ Yale-New Haven Hospital, New Haven, Connecticut; ${ }^{3}$ Department of Internal Medicine, Yale School of Medicine, New Haven, Connecticut.
\end{abstract}

\begin{abstract}
BACKGROUND: Unplanned 30-day hospital readmissions are an important measure of hospital quality and a focus of national regulations. Skilled nursing facilities (SNFs) play an important role in the readmission process, but few studies have examined the factors that contribute to readmissions from SNFs, leaving hospitalists and other hospital-based clinicians with limited evidence on how to reduce SNF readmissions.
\end{abstract}

OBJECTIVE: To understand the perspectives of clinicians working at SNFs regarding factors contributing to readmissions.

DESIGN AND PARTICIPANTS: We prospectively identified consecutive readmissions from SNFs to a single tertiary-care hospital. Index admissions and readmissions were to the hospital's inpatient general medicine service. SNF clinicians who cared for the readmitted patients were identified and interviewed about root causes of the readmissions using a structured interview tool. Transcripts of the interviews were inductively analyzed using grounded theory methodology.

RESULTS: We interviewed 28 clinicians at 15 SNFs. The interviews covered 24 patient readmissions. SNF clinicians described a range of procedural, technological, and cultural contributors to unplanned readmissions. Commonly cited causes of readmission included a lack of coordination between emergency departments and SNFs, poorly defined goals of care at the time of hospital discharge, acute illness at the time of hospital discharge, limited information sharing between a SNF and hospital, and SNF process and cultural factors.

CONCLUSION: SNF clinicians identified a broad range of factors that contribute to readmissions. Addressing these factors may mitigate patients' risk of readmission from SNFs to acute care hospitals. Journal of Hospital Medicine 2017;12:632-638. (@) 2017 Society of Hospital Medicine
Skilled nursing facilities (SNFs) play a crucial role in the hospital readmission process. Approximately 1 in 4 Medicare beneficiaries discharged from an acute care hospital is admitted to a SNF instead of returning directly home. Of these patients, 1 in 4 will be readmitted within 30 days, ${ }^{1}$ a rate significantly higher than the readmission rate of the inpatient population as a whole. ${ }^{2}$ The 2014 Protecting Access to Medicare Act created a value-based purchasing program that will use quality measures to steer funds to, or away from, individual SNFs. When the program takes effect in 2018, the Centers for Medicare \& Medicaid Services will use SNFs' 30-day all-cause readmission rate to determine which SNFs receive payments and which receive penalties. ${ }^{3}$ The Affordable Care Act, passed in 2010, has also established penalties for hospitals with higher than expected readmission rates for Medicare patients. ${ }^{4}$

Despite this intensifying regulatory focus, relatively little is known about the factors that drive readmissions from SNFs. A prospective review of data from SNFs in 4 states has shown that SNFs staffed by nurse practitioners or physician assistants and

\footnotetext{
*Address correspondence and reprint requests: Bennett W. Clark, MD, 600 N. Wolfe St, Baltimore, MD 21287, Telephone: 443-287-3631, Fax: 410-5020923; e-mail: bclark12@jhmi.edu
}

Received: January 23, 2017; Revised: April 12, 2017; Accepted: April 17, 2017

2017 Society of Hospital Medicine DOI 10.12788/jhm.2785 those equipped to provide intravenous therapy were less likely to transfer patients to the hospital for ambulatory care-sensitive diagnoses. ${ }^{5}$ Qualitative studies have provided useful insight into the causes of SNF-to-hospital transfers but have not focused on 30-day readmissions. ${ }^{6,7} \mathrm{~A}$ single survey-based study has examined the causes of SNF-to-hospital readmissions. ${ }^{8}$ However, survey-based methodologies have limited ability to capture the complex perspectives of SNF clinicians, who play a critical role in determining which SNF patients require evaluation or treatment in an acute care setting.

To address this gap in knowledge about factors contributing to SNF readmissions, we conducted a qualitative study examining SNF clinicians' perspectives on patients readmitted to the hospital within 30 days of discharge. We used a structured interview tool to explore the root causes of readmission with frontline SNF staff, with the goal of using this knowledge to inform future hospital quality improvement (QI) efforts.

\section{METHODS}

\section{Case Identification}

Hospital data-tracking software (Allscripts) was used to identify patients who experienced a 30-day, unplanned readmission from SNFs to an academic medical center. We restricted our search to patients whose index admission and readmission were to the medical center's inpatient general medicine service. A study team member (BWC) monitored 
TABLE 1: STRUCTURED INTERVIEW QUESTIONS

NARRATIVE QUESTIONS FROM INTERACT QUALITY IMPROVEMENT TOOL

"Briefly describe the change in condition and other factor(s) that led to the transfer."

"Briefly describe how the [changes in condition] were evaluated and managed."

"In retrospect, does your team think this transfer might have been prevented? Why?"

"In retrospect, does your team think this resident might have been transferred sooner? Why?"

"After review of how this change in condition was evaluated and managed, has your team identified any opportunities for improvement?"

SUPPLEMENTAL QUESTIONS

"How could the hospital system work with you and/or support you to reduce readmissions?"

"How could the hospital system improve its communication with your facility to help prevent readmissions?"

"Does your facility use Epic CareLink [a shared electronic medical record]?"

"Does your facility have a liaison who evaluates patients prior to transfer?"

"Did your staff receive report from a hospital system nurse prior to admitting the patient?"

"Did your staff receive report from a hospital system emergency room nurse after the patient left your facility?"

the dataset on a weekly basis and contacted SNF clinicians by e-mail and telephone to arrange interviews at times of mutual convenience. To mitigate against recall bias, interviews were conducted within 30 days of the readmission in question. A total of 32 cases were identified. No SNF clinicians refused a request for interview. For 8 of these cases, it was not possible to find a time of mutual convenience within the specified 30-day window. The remaining 24 cases involved patients from 15 SNFs across Connecticut. Interviews were conducted from August 2015 to November 2015.

The project was reviewed by our institution's Human Investigation Committee and was exempted from Institutional Review Board review.

\section{Study Participants}

Interviews were conducted on-site at SNFs with groups of 1 to 4 SNF clinicians and administrators. SNF participants were informed of interviewer credentials and the study's QI goals prior to participation. Participation was voluntary and did not affect the clinician's relationship with the hospital or the SNF. Participants were not paid.

\section{DATA COLLECTION}

Interventions to Reduce Acute Care Transfers (INTERACT) is a QI program that includes training for clinicians, communication tools, and advance care planning tools. ${ }^{9}$ INTERACT is currently used in 138 Connecticut SNFs as part of a statewide QI effort funded by the Connecticut State Department of Public Health. In prospective QI studies, ${ }^{10,11}$ implementation of INTERACT has been associated with decreased transfers from SNFs to acute care hospitals. The INTERACT Quality Improvement Tool, one part of the INTERACT bundle of interventions, is a 26-item questionnaire used to identify root causes of transfers from SNFs to acute care hospitals. It includes both checklists and open-ended questions about patient factors, SNF procedures, and SNF clinical decision-making.
We used the INTERACT QI Tool ${ }^{12}$ to conduct structured interviews with nurses and administrators at SNFs. Interviewers used a hard copy of the tool to maintain field notes, and all parts of the questionnaire were completed in each interview. Although the questionnaire elicits baseline demographic and medical information, such as the patient's age and vital signs prior to readmission, the majority of each interview was dedicated to discussion of the open-ended questions in Table 1. Upon completion of the INTERACT QI Tool, the interviewer asked 2 open-ended questions about reducing readmissions and 4 closed-ended questions regarding SNF admission procedures. (Table 1) The supplemental questions were added after preliminary interviews with SNF clinicians revealed concerns about the SNF referral process and about communication between the hospital, emergency department (ED) and SNFs-issues not included in the INTERACT questionnaire. Interviewers used phatic communication, probing questions, and follow-up questions to elicit detailed information from participants, and participant responses were not limited to topics in the questionnaire and the list of supplemental questions.

Interviews were conducted by a hospital clinical integration coordinator, social worker, and a physician $(\mathrm{KB}, \mathrm{MCB}$, $\mathrm{BWC}$ ). All interviewers received formal training in qualitative research methods prior to the study.

All interviews were audio recorded, with permission from the participants, and were professionally transcribed. Field notes were maintained to ensure accuracy of INTERACT QI Tool data. Participant interviews covered no more than two cases per session and lasted from 18 to 71 minutes (mean duration, 38 minutes).

\section{Analysis}

Analysis of transcripts was inductive and informed by grounded theory methodology, in which data is reviewed for repeating ideas, which are then analyzed and grouped to develop a theoretical understanding of the phenomenon under investigation. ${ }^{13,14}$

A preliminary codebook was developed using transcripts of the first 11 interviews. All statements relevant to the readmission process were extracted from the raw interview transcript and collected into a single list. This list was then reviewed for statements sharing a particular idea or concern. Such statements were grouped together under the heading of a repeating idea, and each repeating idea was assigned a code. Using this codebook, each transcript was independently reviewed and coded by three study team members with formal training in inductive qualitative analysis (KB, KTM, BWC). Reviewers assigned codes to sections of relevant text. Discrepancies in code assignment were discussed among the 3 analysts until consensus was reached. Using the method of constant comparison described in grounded theory,the codebook was updated continuously as the process of coding transcripts proceeded. ${ }^{12}$ Changes to the codebook were discussed among the coding team until consensus was achieved. The process of data acquisition and coding continued until theoretical sat- 


\section{TABLE 2: PARTICIPANT CHARACTERISTICS}

\begin{tabular}{lc}
\hline Role - No. (\%) & $11(39)$ \\
Director of nursing & $5(18)$ \\
Nurse manager & $4(14)$ \\
Administrator & $3(11)$ \\
Advanced practice nurse & $3(11)$ \\
Assistant director of nursing & $1(4)$ \\
Infection control nurse & $1(4)$ \\
Staff development coordinator & \\
\hline Educational Attainment - No. (\%) & $18(64)$ \\
Bachelor's degree & $5(18)$ \\
Associate's degree & $5(18)$ \\
Master's degree or higher? & $19.6(3-35$ years) \\
\hline Clinical experience, years - Mean (range) & $3.6(4$ months - 17 years) \\
\hline Time in current position, years - Mean (range) & \\
\hline
\end{tabular}

uration was reached. Themes relating to underlying factors associated with readmissions were then identified based on shared properties among repeating ideas. ATLAS.ti (Scientific Software, Berlin, Germany, Version 7) was used to facilitate data organization and retrieval.

\section{RESULTS}

The SNFs in our study included 12 for-profit and 3 non-profit facilities. The number of licensed beds in each facility ranged from 73 to 360, with a mean of 148 beds. The SNFs had CMS Nursing Home Compare ratings ranging from 1 star, the lowest possible rating, to 5 stars, the highest possible, ${ }^{15}$ with a mean rating of 2.9 stars. Our analysis did not reveal differences in perceived contributions to readmissions from large vs. small or highly rated vs poorly rated SNFs.

Clinicians participating in the interviews came from diverse professional backgrounds. All participating administrators were licensed nurses and continued to provide 1 or more hours of direct patient care per week at the time of the interviews. (Table 2)

The patients in our analysis represented a highly comorbid and medically complex population (Table 3 ). Many had barriers to communication with clinical staff, including non-English-speaking status and underlying dementia.

Five main themes emerged from our analysis: (1) lack of coordination between EDs and SNFs; (2) incompletely addressed goals of care; (3) mismatch between patient clinical needs and SNF capabilities; (4) important clinical information not effectively communicated by hospital; and (5) challenges in SNF processes and culture.

\section{Emergent transitions: Lack of coordination between ED and SNF}

SNF clinicians frequently encountered situations in which a relatively stable patient was readmitted to the hospital after being transferred to the ED, despite the fact that SNF clinicians believed the patient should have returned to the SNF once a specific test was performed or service rendered at the ED. Commonly cited clinical scenarios that resulted in such
TABLE 3: PATIENT CHARACTERISTICS

\begin{tabular}{ll}
\hline $\begin{array}{l}\text { Sex - No.(\%) } \\
\text { Female }\end{array}$ & $14(58)$ \\
\hline Age - yr. (standard deviation) & $74.1(13.4)$ \\
\hline SNF admission status at time of readmission - No.(\%) & \\
Post-Acute Care & $19(79)$ \\
Long-stay & $5(21)$ \\
\hline Comorbid conditions - No.(\%) & \\
Polypharmacy (9 or more medications) & $19(79)$ \\
Heart failure & $10(42)$ \\
Dementia & $9(38)$ \\
Chronic obstructive pulmonary disease & $5(21)$ \\
Surgical complications & $5(21)$ \\
End-stage renal disease & $4(17)$ \\
Cancer, active or under treatment & $3(13)$ \\
\hline Most frequent admission diagnoses - No.(\%) & \\
Acute decompensated heart failure & $3(13)$ \\
Acute kidney injury & $3(13)$ \\
Urinary tract infection & $3(13)$ \\
Pneumonia & $2(8)$ \\
\hline Most frequent readmission diagnoses - No.(\%) & $3(13)$ \\
Acute decompensated heart failure & $3(13)$ \\
Hypoxemic respiratory failure & $2(13)$ \\
Pneumonia & \\
Seizure & \\
\hline
\end{tabular}

readmissions included placement of urinary catheters and evaluation for cystitis. An assistant director of nursing reported that "the ER doesn't want to hear my side of the story," making it difficult for her to provide information that would prevent such readmissions. Other SNF clinicians reported similar difficulties in communicating with ED clinicians.

\section{Code status: Incompletely addressed goals of care}

The SNF clinicians in our study described cases in which patients with end-stage lung disease and disseminated cancer were readmitted to the hospital, despite SNF efforts to prevent readmission and provide palliative care within the SNF. For example, a SNF advanced practice nurse described a case in which a patient with widely metastatic cancer requested readmission to the hospital for treatment of deep vein thrombosis, despite longstanding recommendations from SNF staff that the patient forego hospitalization and enroll in hospice care. After discussion of code status and goals of care with hospital clinicians, the patient chose to enroll in hospice care and not to continue anticoagulation. SNF clinicians often perceived that, in the words of one administrator, "the palliative talks in the hospital outweigh our talks by a lot." Numerous SNF clinicians believed that in-depth clarification of goals of care prior to discharge could prevent some readmissions.

\section{Wrong patient, wrong place: Mismatch between clinical needs and SNF capabilities}

One director of nursing stated that "[when] you read a referral, there's a huge difference sometimes between what you 


\section{TABLE 4: THEMES AND REPRESENTATIVE QUOTES ON READMISSIONS FROM SNFS}

\begin{tabular}{|c|c|c|}
\hline THEME & SUBTHEME & REPRESENTATIVE QUOTES \\
\hline \multirow[t]{3}{*}{$\begin{array}{l}\text { Emergent transitions: } \\
\text { Lack of coordination } \\
\text { between ED and SNF }\end{array}$} & \multirow[t]{3}{*}{$\begin{array}{l}\text { Patient unnecessarily } \\
\text { admitted from ED }\end{array}$} & $\begin{array}{l}\text { Lots of times it might be a facility like ours can't straight cath and need that urologist to scope the patient. We know basically what it is that } \\
\text { the patient needs, but they've gotta go to the emergency room. They've gotta go get evaluated....once the Foley was put back in her, she } \\
\text { could've been sent right back to us. We could've taken care of her after that. It's just the problem of we were unable to put the Foley in. . } \\
\text { that was a [re]admission that really didn't have to happen. (Director of Nursing) }\end{array}$ \\
\hline & & $\begin{array}{l}\text { The ER can get an IV in them, send them back to me, I can manage them here. . . The patient] can stay overnight here, get their first dose } \\
\text { of antibiotics, throw a PICC in in the morning and then send them back and all be under an ER visit or an observation visit and that's simple } \\
\text { enough. What happens a lot of times is ... they admit them because they got to give them antibiotics. That's my biggest frustration. They } \\
\text { don't even call. They don't call the people that they're supposed to be calling, their primary care [doctor], the nursing home. (Director of } \\
\text { Nursing) }\end{array}$ \\
\hline & & $\begin{array}{l}\text { I specifically had a time where I did call the ER and I said to her, "The patient needs a blood transfusion and then send them back." They } \\
\text { didn't do that. They automatically admitted them. A lot of times they are getting admitted for IV antibiotics. We can do that here. They } \\
\text { don't need to stay for a UTI. (Nurse Manager) }\end{array}$ \\
\hline
\end{tabular}

ED does not update SNF I've been here 10 years. They never call us, give us updates about how the patient is doing. (Assistant Director of Nursing) on patient status

\begin{tabular}{ll}
\hline ED culture hostile & I feel like the ER doesn't want to hear my side of story. [They] say, "Don't call me. Don't message me. He's still not here. I'm pretty sure \\
to communication from & $\begin{array}{l}\text { we'll evaluate him here." (Assistant Director of Nursing) } \\
\text { SNF }\end{array}$ \\
& $\begin{array}{l}\text { I've stopped calling [the ER] because it doesn't work. . I got three very impatient nurses that hung up on me twice and then finally trans- } \\
\text { ferred me to a charge nurse in the ER who couldn't get me off the phone fast enough and told me she had all the information she needed } \\
\text { before I finished talking. (Administrator) }\end{array}$
\end{tabular}

\section{Code status:}

Incompletely defined goals of care
Goals of care not addressed at hospital

[Regarding a patient with glioblastoma multiforme and acute DVT]: I think she was really sick, and I think a lot of it was the family dynamic and them really pushing for hospitalization. . . I've been here for two and a half years. I've never sent anybody out for a DVT. We just treat here. I think that more palliative talks prior to her coming here from oncology in the hospital. . . maybe that would have prevented this readmission if we could treat more palliatively. Because she ended up going to hospice. (Advanced Practice Nurse)

Some of those folks that we're sending out, we might have been able to keep here with goals of care of keeping them comfortable and so forth rather than the revolving door. (Administrator)

Family suspicious of SNF clinician's

The residents that I can think of that we've had issues with regarding a short turnaround time- - they've come back to us and we've had to turn them back around - usually, that relates to care decisions and goals of care, palliative care conversations being had at the hospital. We have those on a constant basis here. If we send someone out to the hospital, and their decline is such that that conversation needs to be revisited and maybe from a little different perspective because it's the hospital and not the nursing home, the families perceive that differently. (Administrator)

I know working in long-term care or short-term rehab, these facilities, the palliative talks in the hospital outweigh our talks by a lot. If the faml If [the family] at least heard it from the hospitalist, it's weighted more. (Administrator)

Sometimes. . . family wants everything, everything, everything. [The patient] is basically dying. Then we send them to the hospital and the family decides to change their mind. (Assistant Director of Nursing)

Wrong patient, wrong place: Mismatch between patient clinical needs and SNF capabilities available at SNF
Necessary treatment and He was respiratory compromised from day one of admission [to our SNF]. We were monitoring his oxygen levels and requiring nebulizers. medical personnel not He would de-sat, need a mask, need a nasal cannula, rest. Could not participate in therapy without de-sating upon exertion. . .

he was just really compromised respiratory-wise. (Nurse Manager)

I think insurance has a lot to do with it too now. They're pushing them out of the hospital a lot quicker. . . sometimes, unfortunately, patients aren't stable. CHF, you can go home and go back to the hospital, you can come [to a SNF] and go back to the hospital. (Nurse Manager)

The diagnostic [tests] can get done so much quicker in a hospital. We can take a urine right now. They won't pick it up until tomorrow morning. If I get urine from somebody five minutes after the lab's left, I have to wait until the next day for them to come pick that up, so that delays that for another day. (Advanced Practice Nurse)

Interviewer: Do you have EKGs in-house?

Interviewee: Not in house, but I can get one stat. But not that stat. (Assistant Director of Nursing) read and what you see." SNF clinicians reported that this discrepancy between clinical report and clinical reality often leads to patients being placed in SNFs that are unequipped to care for them. Many patients were perceived as being too ill for discharge from the acute-care setting in the first place. A nurse manager described this as a pattern of "pushing patients out of the hospital." However, mismatches in clinical disposition were also seen as contributing to readmissions for medically stable patients, such as those with dementia, for whom SNFs frequently lack adequate staffing and physical safeguards.

\section{Missing links: Important clinical information not effectively communicated by hospital}

SNF clinicians described numerous challenges in formulating plans of care based on hospital discharge documentation.
Discrepancies between discharge summaries and patient instructions were perceived as common and potential causes of readmissions. For patients discharged from the academic medical center in this study, medication instructions are included in both the discharge summary sent to the SNF and in a patient instruction packet. Several SNF clinicians said that it was common for a course of antibiotics to be listed on the discharge summary but not the patient instruction packet, or vice versa. SNF clinicians, who usually lack access to the hospital's electronic medical record, have limited means for determining the correct document. Other important clinical data points, such as intermittent intravenous (IV) furosemide dosing and suppressive antibiotic regimens, were omitted from discharge paperwork altogether. SNF clinicians had difficulty reaching hospital clinicians who could clarify these clinical questions. "Good luck finding the per- 


\section{TABLE 4: THEMES AND REPRESENTATIVE QUOTES ON READMISSIONS FROM SNFs (continued)}

\begin{tabular}{|c|c|c|}
\hline THEME & SUBTHEME & REPRESENTATIVE QUOTES \\
\hline & $\begin{array}{l}\text { Necessary support } \\
\text { staff and physical } \\
\text { infrastructure not } \\
\text { available at SNF }\end{array}$ & $\begin{array}{l}\text { We have a gentleman we just sent out today. } 15 \text { doors wouldn't keep him in. He's obsessed with going home. When you talk to him, he's a } \\
\text { lovely gentleman, but every-"I want to go home. Call my son. Is my son on the way? I want to go home." He's prying doors open, offering } \\
\text { people } \$ 5.00 \text { to take him home. A locked unit [would have been] very different. (Administrator) }\end{array}$ \\
\hline \multirow[t]{8}{*}{$\begin{array}{l}\text { Missing links: } \\
\text { Important clinical information } \\
\text { not communicated by hospital }\end{array}$} & $\begin{array}{l}\text { Medical plan not com- } \\
\text { municated } \\
\text { to SNF by hospital }\end{array}$ & $\begin{array}{l}\text { We just had a patient come in who used to get IV Lasix twice a week, and we weren't updated on that. (Advanced Practice Nurse) } \\
\text { You can see they printed [the summary] out, maybe at } 9 \text { a.m. Then the patient gets here at } 4 \text { p.m. Maybe there's no pain meds. Maybe } \\
\text { there's no antibiotic, but then there's a discharge summary that says, oh, yeah. We put 'em on this. (Nurse Manager) }\end{array}$ \\
\hline & $\begin{array}{l}\text { Hospital culture } \\
\text { hostile to } \\
\text { communication }\end{array}$ & $\begin{array}{l}\text { Sometimes you have a very specific nursing question that you just want to talk to another nurse about, and we had actually someone } \\
\text { complain. .. [One of our nurses] wasn't allowed to ask the nurse the question she asked. The discharge planner decided it was a stupid } \\
\text { question that she shouldn't ask. Then we were all told, "Don't talk to the nurses. Don't call the hospital." (Director of Nursing) }\end{array}$ \\
\hline & & $\begin{array}{l}\text { Let's say a patient comes to us with MRSA. If you call [the hospital] again and ask them where was the source of infection, they don't } \\
\text { know. They say, "We don't know. We don't know." (Infection Control Nurse) }\end{array}$ \\
\hline & $\begin{array}{l}\text { Hospital clinician } \\
\text { not available } \\
\text { to discuss patient }\end{array}$ & $\begin{array}{l}\text { It's a lot of calls back and forth, and sometimes it's a couple days after. Good luck finding the person that took care of [the patient] three } \\
\text { days before. (Director of Nursing) }\end{array}$ \\
\hline & $\begin{array}{l}\text { Hospital report } \\
\text { does not match } \\
\text { clinical reality }\end{array}$ & $\begin{array}{l}\text { When you read a referral, there's a huge difference sometimes between what you read and what you see, and it goes both ways. } \\
\text { Sometimes they look terrible on paper, and you take them, and they really don't look that bad. Other times they look pretty simple, pretty } \\
\text { straightforward, and that's not what you find when they are actually in. (Director of Nursing) }\end{array}$ \\
\hline & $\begin{array}{l}\text { Incomplete or erroneous } \\
\text { discharge information }\end{array}$ & $\begin{array}{l}\text { A lot of times you get all these lists of meds. There's a once a week thing, you don't know if it was given, if they gave it there or when they } \\
\text { give it, when's the last time someone had it. (Nurse Manager) }\end{array}$ \\
\hline & & $\begin{array}{l}\text { I find when you get a report it's really like- - I don't know. What's the word l'm looking for? Useless. They are all over the place. They don't } \\
\text { know what they're talking about. I just took care of this patient. The patient's been there for weeks. Stuff you really need to know you } \\
\text { don't get. A lot of it—-then it doesn't come over with the paperwork too. (Staff Development Coordinator) }\end{array}$ \\
\hline & & $\begin{array}{l}\text { Either [the discharge summaries] are filled with just a bunch of mumbo-jumbo that means absolutely nothing, like it's not related to why we } \\
\text { sent them out, or literally there's just nothing, absolutely nothing. (Assistant Director of Nursing) }\end{array}$ \\
\hline \multirow[t]{6}{*}{$\begin{array}{l}\text { Change starts at home: } \\
\text { Challenges in SNF processes } \\
\text { and culture }\end{array}$} & $\begin{array}{l}\text { Reduced weekend, } \\
\text { holiday staffing }\end{array}$ & $\begin{array}{l}\text { If we get an admission on Friday that patient's probably not going to be seen by a medical doctor until Monday. If we don't have all those } \\
\text { things in order, then something may be missed. The potential of the readmission is much greater, especially with Friday admissions. } \\
\text { (Director of Nursing) }\end{array}$ \\
\hline & $\begin{array}{l}\text { "When in doubt, } \\
\text { send them out" }\end{array}$ & $\begin{array}{l}\text { We are working very, very hard to transfer people less. It's a big buy-in with a lot of the doctors. . . We're trying to educate the doctors more } \\
\text { and more, and the nurses, on what we can do here versus sending [the patient] out. (Director of Nursing) }\end{array}$ \\
\hline & & $\begin{array}{l}\text { We probably could have treated more inhouse... [the patient's] speech was a little bit more slurred. The oncall [doctor] was concerned } \\
\text { with possible stroke, and so we didn't want to take that chance. Otherwise, we could have handled stuff more inhouse. (Nurse Manager) }\end{array}$ \\
\hline & $\begin{array}{l}\text { Patient's family } \\
\text { prefers readmission }\end{array}$ & $\begin{array}{l}\text { The families don't understand what's going on. . . The cultural changes, this generation of people that are in the Medicare years were } \\
\text { taught to go to the ER if you have a problem. . . When in doubt send them out. It was a motto. People still think, "This is a nursing home. } \\
\text { They can't do anything." We say, "Hey. We're going to give you some IV Lasix." "Oh my God. You're going to do that here?" (Advanced } \\
\text { Practice Nurse) }\end{array}$ \\
\hline & & $\begin{array}{l}\text { We have some families that aren't always comfortable with our extended abilities. They have the old view of what the facility is capable of } \\
\text { doing. (Director of Nursing) }\end{array}$ \\
\hline & $\begin{array}{l}\text { Covering clinician } \\
\text { unfamiliar with patient }\end{array}$ & $\begin{array}{l}\text { I don't know if there's quite the good handoff between the doctors that are on-call and the doctors that are here. We have } 300 \text { patients. } \\
\text { How many doctors cover for how many doctors? There could be a thousand patients they're covering for. That's their end, something they } \\
\text { can work on better. (Administrator) }\end{array}$ \\
\hline
\end{tabular}

son that took care of [the patient] three days before," said one director of nursing.

\section{Change starts at home: Challenges in SNF processes and culture}

Many clinicians in our study reported that their facilities had recently added clinical capabilities in an effort to care for patients with complex medical problems. For example, to prevent transfers of patients with decompensated heart failure, several facilities in our study had recently obtained certification to give IV diuretics. However, as one director of nursing stated, these efforts require "buy-in" from doctors to decrease readmissions. That buy-in has not always been forthcoming. SNF clinicians also reported difficulty convincing patients and families that their facilities are capable of providing care that, in the past, might only have been available in acute-care settings.
These themes, along with associated sub-themes and representative quotations, are shown above (Table 4).

\section{DISCUSSION}

Our study suggests that the interaction between EDs and SNFs is an important and understudied domain in the spectrum of events leading to readmission. Prior studies have documented inadequacies in patient information provided by SNFs to EDs. ${ }^{16,17}$ Efforts to improve SNF-to-ED information sharing have focused on making sure that ED clinicians have important baseline information about patients transferred from a SNF. ${ }^{18,19}$ However, many of the clinicians in our study reported taking proactive steps to communicate with ED clinicians. These efforts encountered logistical and cultural barriers, with information that might have prevented readmission failing to reach ED providers. Many of 
the SNF clinicians in our study perceived this failure as a common cause of readmission, especially for relatively stable SNF patients.

Previous studies have pointed to a role for goals of care discussions in reducing hospital readmissions. ${ }^{20}$ Our data underscore an important qualification to these findings: Location matters. The SNF clinicians in our study reported frequent and detailed goals of care discussions with their patients. However, they also reported that goals of care discussions held in the subacute setting carried less weight with patients and families than discussions held in the hospital. SNF clinicians described a number of cases in which patients were willing to adjust code status or goals of care only after being readmitted to the hospital.

Our study also points to the implications of existing research showing that patients are discharged from acute care hospitals "quicker and sicker" than they had been prior to the 1983 adoption of Medicare's prospective payment system. ${ }^{21}$ Specifically, the SNF clinicians we interviewed perceived a strong link between patient acuity at the time of transfer and SNFs' persistently high readmission rates. As SNFs have worked to expand their clinical capabilities, they struggle to win buy-in from physicians and families, many of whom view SNFs as incapable of managing acute illness. Many SNF clinicians also pointed to deficiencies in their own referral and admission processes as a recurring cause of readmissions. For example, several patients in our analysis suffered from dementia. Although these patients were stable enough to leave the acute care setting, the SNF clinicians responsible for their readmissions felt that their SNFs were not well-equipped to care for patients with dementia and that the patients should instead have been transferred to facilities with more robust resources for dementia care.

Finally, our findings highlight a fundamental tension between hospitals and SNFs: Which facility ought to shoulder the responsibility and cost for services that may prevent a readmission - the hospital or the SNF? For example, does responsibility for coordinating subspecialist evaluation of a patient's chronic condition fall to the hospital or to the SNF? If such an evaluation is undertaken during a hospitalization, it prolongs the patient's hospital stay and happens at the hospital's expense. If the patient is discharged to a SNF and sees the subspecialist in clinic, then the SNF must pay for transportation to and from the clinic appointment. SNF clinicians expressed near unanimity that fragmented models of care and high barriers to communication made it difficult to design solutions to these dilemmas.

\section{Strengths and limitations}

To our knowledge, this is the first interview-based study examining SNF clinicians' perspectives on unplanned, 30day hospital readmissions. We gathered information from clinicians with a range of clinical experience, all of whom had cared directly for the patient who had been readmitted. Our data came from clinicians at 15 SNFs of varying sizes and quality ratings, allowing us to identify a broad range of factors contributing to readmissions.

Because this study relied on qualitative methods, it should be viewed as hypothesis-generating rather than hypothesis-confirming. Further research is needed to determine whether variables related to the themes above are causally linked to SNF readmissions. We identified cases for review using convenience sampling of a cohort of readmitted patients at a single tertiary-care hospital, and all participating SNFs were located in Connecticut. These factors may limit the generalizability of our findings. Although the clinicians we interviewed occupied diverse roles within their respective SNFs, our sample did not include direct-care staff without managerial responsibility, such as certified nursing assistants or licensed practical nurses. This prevented our study from identifying themes into which managers would have limited insight, especially those involving cultural and management practices leading to poor communication between them and their staff. Because our study examines cases in which discharge and readmission were to a general medicine service, it may not describe factors relevant to patients discharged from subspecialist or surgical services.

\section{Implications for future QI efforts and research}

Several clinicians we interviewed suggested that readmissions might be reduced by dedicating the services of a hospital professional, such as a nurse or case manager, to monitoring the clinical course of medically complex patients after discharge. A dedicated "transition coach" could clarify deficiencies in discharge paperwork, facilitate necessary follow-up appointments, liaise with staff at both the hospital and the SNF, or coordinate acquisition of necessary equipment. Prospective trials have demonstrated that such interventions can decrease readmission rates among hospitalized patients, ${ }^{22,23}$ but formal studies have not been carried out among cohorts of SNF patients.

Prior efforts to improve SNF-ED information sharing have focused on making sure that ED clinicians have important baseline information about patients transferred from a SNF. ${ }^{24,25}$ The experiences of SNF clinicians in our study suggest that important information also fails to make its way from ED providers to SNFs and that this failure results in unnecessary readmissions of relatively stable SNF patients. Thus, hospitals may be able to prevent SNF readmissions by creating lines of communication between EDs and SNFs and by ensuring that ED physicians and mid-level providers are familiar with the clinical capabilities of local SNFs.

Future research and QI work should also investigate approaches to care coordination that ensure that complex patients are placed in SNFs with resources adequate to address their comorbidities. Potential interventions might include increased use of SNF "liaisons," who would evaluate patients in-person prior to approving transfer to a given SNF. As has been previously suggested, ${ }^{26}$ hospitals might also reduce readmissions by narrowing the pool of facilities to which they transfer patients, thereby building more robust, interconnected relationships with a smaller number of SNFs. 


\section{CONCLUSION}

SNF clinicians identified areas for improvement at almost every point in the chain of events spanning hospitalization, discharge, and transfer. Among the most frequently cited contributors to readmissions were clinical instability at the time of discharge and omission of clinically important information from discharge documentation. Improved communication between hospitals, ED clinicians, and SNFs, as well as more thoroughly defined goals of care at the time of discharge, were seen as promising ways of decreasing readmissions. Successful interventions for reducing readmissions from SNFs will likely require multifaceted approaches to these problems.

Disclosure: This research was supported by a grant (\#P3OHS023554-01) from the Agency for Healthcare Research and Quality (AHRQ) and received support from Yale New Haven Hospital and the Claude D. Pepper Older Americans Independence Center at Yale University School of Medicine (\#P30AG021342 NIH/NIA).

\section{REFERENCES}

1. Mor $\mathrm{V}$, Intrator $\mathrm{O}$, Feng $\mathrm{Z}$, et al. The revolving door of rehospitalization from skilled nursing facilities. Health Aff. 2010;29(1):57-64.

2. Department of Health and Human Services. Medicare.gov Hospital Compare. https://medicare .gov/hospitalcompare/compare. Accessed October 21, 2015.

3. Centers for Medicare and Medicaid Services. Proposed fiscal year 2016 payment and policy changes for Medicare Skilled Nursing Facilities. https://cms.gov. Accessed October 21, 2015

4. The Patient Protection and Affordable Care Act: Detailed Summary. Democratic Policy and Communications Committee website. http://www.dpc.senate.gov/ healthreformbill/healthbill04.pdf. Accessed August 22, 2016.

5. Intrator $\mathrm{O}$, Zinn J, Mor V. Nursing home characteristics and potentially prevent able hospitalizations of long-stay residents. J Am Geriatr Soc. 2004;52:1730-1736.

6. Ouslander JG, Lamb G, Perloe M, et al. Potentially avoidable hospitalizations of nursing home residents: frequency, causes and costs. J Am Geriatr Soc. 2010;58:627-635.

7. Lamb G, Tappen R, Diaz S, et al. Avoidability of hospital transfers of nursing home residents: perspectives of frontline staff. J Am Geriatr Soc. 2011;59:16651672.

8. Ouslander JG, Naharci I, Engstrom G, et al. Hospital transfers of skilled nursing facility (SNF) patients within 48 hours and 30 days after SNF admission. J Am Med Dir Assoc. 2016; doi: 10.1016/j.jamda.2016.05.021.

9. Ouslander JG, Lamb G, Tappen R et al. Interventions to reduce hospitalizations from nursing homes: Evaluation of the INTERACT II collaborative quality improvement project. J Am Geriatr Soc. 2011; 59:745-753.

10. Ouslander JG, Perloe M, Givens JH et al. Reducing potentially avoidable hospitalization of nursing home residents: Results of a pilot quality improvement project. J Am Med Dir Assoc. 2009; 10:644-652.

11. Tena-Nelson R, Santos K, Weingast E et al. Reducing preventable hospital transfers: Results from a thirty nursing home collaborative. J Am Med Dir Assoc. 2012; 13:651-656.

12. Florida Atlantic University. Interventions to Reduce Acute Care Transfers. https://interact2.net/docs/INTERACT\%20Version\%204.0\%20Tools/INTERACT\%204.0\%20NH\%20Tools\%206_17_15/148604\%20QI_Tool\%20for\%20 Review\%20Acute\%20Care\%20Transf_AL.pdf

13. Oktay, Julianne. Grounded Theory. New York: Oxford University Press, 2012.

14. Auerbach, Carl and Silverstein, Louise B. Qualitative Data. New York: NYU Press, 2003.

15. Department of Health and Human Services. Medicare.gov Nursing Home Compare. https://medicare .gov/nursinghomecompare. Accessed April 4, 2016.

16. Jones JS, Dwyer PR, White LJ, et al. Patient transfer from nursing home to emergency department: outcomes and policy implications. Acad Emerg Med. 1997 Sep;4(9):908-15.

17. Lahn M, Friedman B, Bijur P, et al. Advance directives in skilled nursing facility residents transferred to emergency departments. Acad Emerg Med. 2001 Dec;8(12):1158-62.

18. Madden C, Garrett J, Busby-Whitehead J. The interface between nursing homes and emergency departments: a community effort to improve transfer of information. Acad Emerg Med. 1998 Nov;5(11):1123-6.

19. Hustey FM, Palmer RM. An internet-based communication network for information transfer during patient transitions from skilled nursing facility to the emergency department. J Am Geriatr Soc. 2010 Jun;58(6):1148-52.

20. O'Connor N, Moyer ME, Behta M, et al. The Impact of Inpatient Palliative Care Consultations on 30-Day Hospital Readmissions. J Pall Med. 2015 Nov 1; 18(11):956-961.

21. Qian X, Russell LB, Valiyeva E, et al. "Quicker and sicker" under Medicare's prospective payment system for hospitals: new evidence on an old issue from a national longitudinal survey. Bull Econ Res. 2011;63(1):1-27.

22. Naylor MD, Brooten DA, Campbell RL, Maislin G, McCauley KM, Schwartz JS. Transitional care of older adults hospitalized with heart failure: a randomized, controlled trial. J Am Geriatr Soc. 2004 May;52(5):675-84.

23. Coleman EA, Parry C, Chalmers S, Min SJ. The care transitions intervention: results of a randomized controlled trial. Arch Intern Med. 2006 Sep 25;166(17): $1822-1828$.

24. Madden C, Garrett J, Busby-Whitehead J. The interface between nursing homes and emergency departments: a community effort to improve transfer of information. Acad Emerg Med. 1998 Nov;5(11):1123-6.

25. Hustey FM, Palmer RM. An internet-based communication network for information transfer during patient transitions from skilled nursing facility to the emergency department. J Am Geriatr Soc. 2010 Jun;58(6):1148-52.

26. Rahman M, Foster AD, Grabowski DC, Zinn JS, Mor V. Effect of hospital-SNF referral linkages on rehospitalization. Health Serv Res. 2013 Dec;48(6 Pt 1):1898-919. 